\title{
Stable articulatory tasks and their variable formation: Tamil retroflex consonants
}

\author{
Caitlin Smith ${ }^{1}$, Michael Proctor ${ }^{2}$, Khalil Iskarous ${ }^{1}$, Louis Goldstein ${ }^{1}$, Shrikanth Narayanan ${ }^{3}$ \\ ${ }^{1}$ Department of Linguistics, University of Southern California, Los Angeles, CA, USA \\ ${ }^{2}$ Department of Linguistics, University of Western Sydney, Sydney, NSW, Australia \\ ${ }^{3}$ Department of Electrical Engineering, University of Southern California, Los Angeles, CA, USA \\ smithcm@usc.edu, michael.proctor@uws.edu.au, kiskarou@usc.edu, louisgol@usc.edu, \\ shriasipi.usc.edu
}

\begin{abstract}
A real-time MRI examination of retroflex stops and rhotics in Tamil reveals that in some contexts these consonants may in fact be achieved with little or no retroflexion of the tongue tip. Rather, maneuvering and shaping of the tongue in order to achieve post-alveolar contact varies across vowel contexts. Between back vowels /a/ and /u/, post-alveolar constriction involves curling back of the tongue tip, but in the context of high front vowel $/ \mathrm{i} /$, the same constriction is achieved by bunching of the tongue. It appears that though there is a stable constriction target in the post-alveolar region, its achievement is not fixed but is instead a consequence of the variable state of the vocal tract in different vowel contexts. Articulatory configurations of the tongue across these vowel contexts were examined by comparing measures of Gaussian curvature at evenly spaced points along the vocal tract. The results support the notion that so-called retroflex consonants have a specified target constriction in the post-alveolar region, but that the specific articulations employed to achieve this constriction are not fixed, in keeping with the task dynamic model of speech production.
\end{abstract}

Index Terms: retroflex, Tamil, real-time MRI

\section{Introduction}

The class of retroflex consonants is broadly identified by a curling back of the tongue tip in order to achieve contact with the palate in the post-alveolar or palatal region [1]. However, it is unclear whether this tongue curling is actively controlled or merely a consequence of the state of the vocal tract during the achievement of consonant constriction. These two possible control regimes make different predictions regarding the stability of tongue maneuvering and shaping of retroflex consonants across vowel contexts, in which the tongue body is engaged in different ways. If curling of the tongue is a controlled maneuver of a retroflex consonant, we might expect tongue shaping to be stable across vowel contexts. However, if it is only the constriction location that is controlled, we can expect variation in how that constriction is achieved due to the differing state of the vocal tract across vowel contexts.

In order to test these predictions, we look to the Dravidian language Tamil, whose phoneme inventory includes a retroflex stop, nasal, rhotic, and lateral [2]. Using real-time MRI we will examine whether there is uniform maneuvering in the production of retroflex consonants across vowel contexts, or whether these consonants are merely specified for constriction location, with no global control over tongue maneuvering and shaping. This study will also examine the stability of constriction location across vowel contexts as confirmation that the constriction task remains the same while tongue shaping may vary.

Much previous work on retroflex consonants focuses on cross-linguistic variation in their production, with little discussion of within-language variation caused by the environment in which a retroflex is produced. One study describes Tamil's retroflex stop as a sub-apical palatal closure with the tongue curled quite far back, in contrast with the articulation of retroflexes in other languages, such as Hindi's less curled and more anterior retroflex [3]. Whether the Tamil retroflex's curling and posterior place of articulation hold across environments is not discussed. It is true that crosslinguistic variation in the production of what are broadly referred to as retroflexes can tell us generally that different languages may have different goals of production for these consonants. However, here we aim to discover what that goal of production is for retroflexes of Tamil by examining which aspects of their articulation vary and which remain stable across environments.

A previous EMA study on Tamil consonants found that retroflex consonants were more variable in tongue tip orientation than dental and alveolar consonants [4]. Our use of real-time MRI will allow us to discover the source of this variability as we examine shaping and maneuvering along the entire vocal tract as it unfolds over time. Static MRI has previously been used to examine tongue shaping during Tamil retroflexes, though constrictions were sustained for a period of several seconds and thus were not embedded in varying vowel contexts [5].

\section{Methods}

\subsection{Target items}

Target consonants were produced in symmetrical vowel contexts $/ \mathrm{a} / \mathrm{h} / \mathrm{i} /$, and $/ \mathrm{u} /$. All elicited items were nonsense words of the form $/ \mathrm{pV} \quad \mathrm{Vm} /$ and were produced in isolation. Items were presented in Tamil orthography, which distinguishes between dental/alveolar and retroflex consonants. Dental/alveolar and retroflex stops, nasals, and rhotics were targeted, as well as an alveolar lateral approximant. Speakers 1, 2, and 3 produced five repetitions of each target item, while Speaker 4 produced three repetitions. Here, we will only report on the results of our analysis of the retroflex stop and rhotic.

\subsection{Image acquisition}

Midsagittal images of the four subjects' vocal tracts were acquired using a real-time MRI protocol developed for the examination of speech production [6]. Each subject lay on his or her back with head stabilized throughout the scan. The field 
of view for each image is $200 \mathrm{~mm} \times 200 \mathrm{~mm}$ including the glottis, pharynx, and oral and nasal cavities, with a resolution of $68 \times 68$ pixels. Images were reconstructed at a rate of 33.18 frames per second.

\subsection{Analysis}

Tongue and palate edge tracking was performed using a custom graphical user interface in MATLAB [7], [8]. A polarrectangular grid of approximately thirty lines orthogonal to the vocal tract was laid over the video images. For each frame of video, the air/tissue boundaries for the upper and lower surfaces of the vocal tract were automatically detected at each gridline and corrected manually if necessary (see Figure 1).

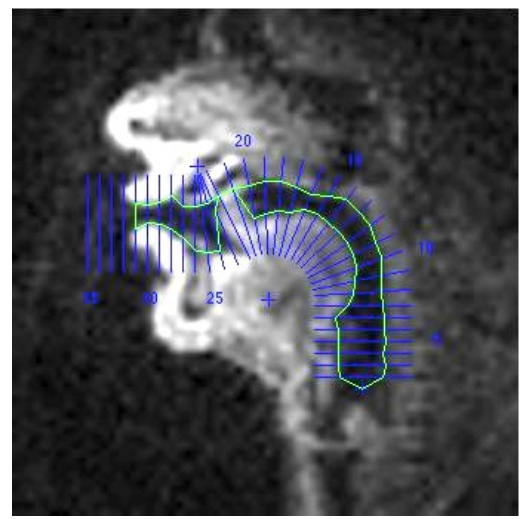

Figure 1: Frame of tongue edge-tracked MRI video with overlaid analysis grid

Because the constriction for retroflex consonants is considerably more posterior at the initial achievement of constriction and more anterior at the release of constriction, all measures were taken at the initial point of contact between the tongue and the palate. Video frames corresponding to the achievement of target constriction for each consonant were identified. At this frame, measures for constriction location and tongue shaping/curvature were taken.

Constriction location was measured by the gridline at which there was contact between the tongue and the upper surface of the vocal tract. Tongue shaping was measured using a calculation of Gaussian curvature. First, the outline of the lower surface of the vocal tract was down-sampled to fifteen evenly spaced points. For each set of three adjacent points, the inverse of the diameter of the circle passing through these three points was calculated and then multiplied by 100 . This curvature score was measured as negative whenever the center of that circle lay outside of the plane of the tongue curvature, corresponding to tongue curling.

\section{Results}

\subsection{Constriction location}

Within-subject mean gridline of consonant constriction for the retroflex stop and rhotic in each vowel context are reported in Tables 1 and 2. For the most part, constriction location across vowel contexts did not vary by more than one gridline (though Subject 1 showed somewhat greater variability). In the post-alveolar region, one gridline corresponds to approximately six millimeters. Note that gridlines are counted beginning at the glottis; therefore, a higher gridline number corresponds to a more anterior constriction location. Also note that because each subject's analysis grid is individually fit to his or her unique vocal tract, it is not possible to compare constriction location as measured by gridline across speakers.

Table 1. Within-speaker constriction location for retroflex stop in each vowel context

\begin{tabular}{cccc}
\hline & \multicolumn{3}{c}{ Mean Constriction Gridline } \\
Speaker & /a/ Context & /u/ Context & /i/ Context \\
\hline $\mathbf{1}$ & 26.4 & 26.8 & 29 \\
$\mathbf{2}$ & 21.2 & 21.4 & 21.6 \\
$\mathbf{3}$ & 20.6 & 20.8 & 21 \\
$\mathbf{4}$ & 24 & 24.7 & 25.7 \\
\hline
\end{tabular}

Table 2. Within-speaker constriction location for retroflex rhotic in each vowel context

\begin{tabular}{cccc}
\hline & \multicolumn{3}{c}{ Mean Constriction Gridline } \\
Speaker & /a/ Context & /u/ Context & /i/ Context \\
\hline $\mathbf{1}$ & 28.6 & 26 & 28 \\
$\mathbf{2}$ & 21 & 21 & 21.2 \\
$\mathbf{3}$ & 19 & 19.6 & 19.2 \\
$\mathbf{4}$ & 23.7 & 23.7 & 24.3 \\
\hline
\end{tabular}

\subsection{Tongue shaping}

The number of tokens with negative curvature along the tongue blade for the retroflex stop and rhotic are reported in Tables 3 and 4 . Recall that Subject 4 only produced three repetitions of each target consonant.

Table 3. Number of tokens with negative curvature scores along tongue blade/body region for retroflex stop in each vowel context

\begin{tabular}{|c|c|c|c|}
\hline \multirow[t]{2}{*}{ Speaker } & \multicolumn{3}{|c|}{ Number of Negative Curvature Scores } \\
\hline & /a/ Context & $/ \mathrm{u} /$ Context & /i/ Context \\
\hline 1 & 5 & 5 & 3 \\
\hline 2 & 4 & 5 & 2 \\
\hline 3 & 5 & 5 & 0 \\
\hline 4 & 3 & 3 & 2 \\
\hline
\end{tabular}

Table 4. Number of tokens with negative curvature scores along tongue blade/body region for retroflex rhotic in each vowel context

\begin{tabular}{|c|c|c|c|}
\hline \multirow[t]{2}{*}{ Speaker } & \multicolumn{3}{|c|}{ Number of Negative Curvature Scores } \\
\hline & /a/ Context & /u/ Context & /i/ Context \\
\hline 1 & 5 & 5 & 4 \\
\hline 2 & 5 & 5 & 2 \\
\hline 3 & 5 & 5 & 2 \\
\hline 4 & 3 & 3 & 1 \\
\hline
\end{tabular}

Every subject showed a greater tendency toward negative curvature in the $/ \mathrm{a} /$ and $/ \mathrm{u} /$ vowel contexts than in the $/ \mathrm{i} /$ context for both stops and rhotics. The absence of any negative curvature along the tongue blade for some tokens produced in the /i/ context suggests that some kind of maneuver other than retroflexion is being performed. Figure 2 demonstrates what precisely is occurring during individual tokens of the production of a retroflex stop in back versus front vowel 
contexts that would lead to the presence or absence of negative curvature along the tongue blade.

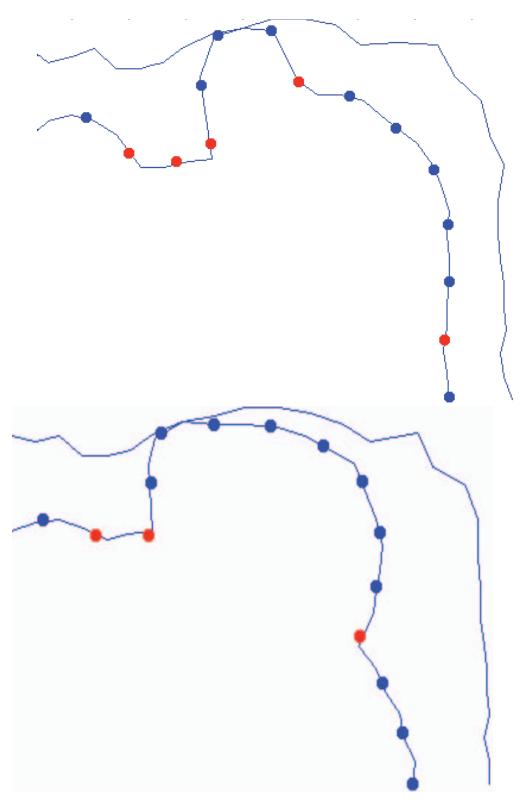

Figure 2: Above: outline of upper and lower surfaces of vocal tract during production of retroflex stop in / $/$ / context. Below: outline of upper and lower surfaces of vocal tract during production of retroflex stop in lal

context. Blue indicates positive curvature; red indicates negative curvature.

The token produced in the /a/ context has one point along the tongue blade which received a negative curvature score of -4.14 . It is at this point that the curling back of the tongue tip occurs. In contrast, for the token produced in the /i/ context we can see that there is no negative curvature along the tongue blade; the lowest tongue blade curvature score for this token is 1.05. Rather than seeing a curling back of the tongue tip, the tongue appears bunched.

Minimum curvature scores along the tongue blade were collected and averaged across tokens, speakers, and consonant type (stop or rhotic). The results of these calculations are reported in Figure 3.

\section{- $/ \mathrm{a} /$ Context $\quad \square / \mathrm{u} /$ Context $\quad$ - /i/Context}

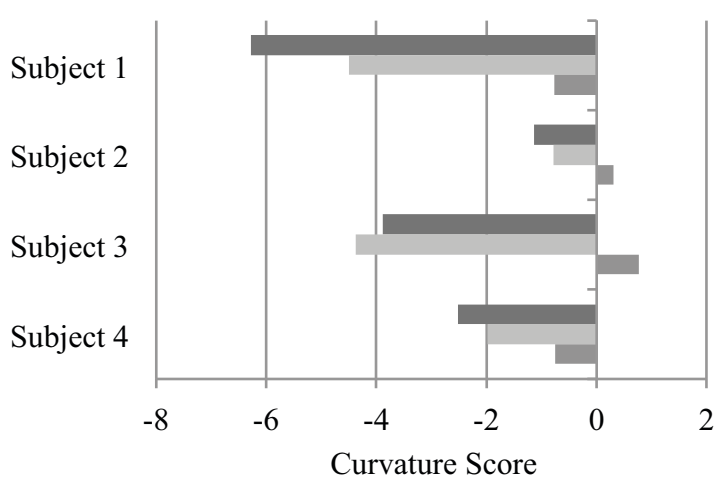

Figure 3: Mean minimum curvature scores along tongue blade region for retroflex consonants in each vowel context
Table 5. Mean curvature scores along tongue blade/body region for retroflex consonants in each vowel context

\begin{tabular}{cccc}
\hline \multirow{2}{*}{ Speaker } & \multicolumn{3}{c}{ Mean Curvature Scores } \\
& /a/ Context & /u/ Context & /i/ Context \\
\hline $\mathbf{1}$ & -6.27 & -4.50 & -0.77 \\
$\mathbf{2}$ & -1.14 & -0.79 & 0.30 \\
$\mathbf{3}$ & -3.88 & -4.37 & 0.77 \\
$\mathbf{4}$ & -2.52 & -1.98 & -0.76 \\
\hline Mean & $\mathbf{- 3 . 5 6}$ & $\mathbf{- 3 . 0 1}$ & $\mathbf{- 0 . 0 4 4}$ \\
\hline
\end{tabular}

Mean curvature scores along the tongue blade appear to vary across vowel contexts, with consonants produced in back vowel contexts $/ \mathrm{a} / \mathrm{and} / \mathrm{u} / \mathrm{showing}$ a greater degree of negative curvature than those produced in high front vowel /i/ context. In particular, note that the average curvature scores for Subjects 2 and 3 are actually positive for retroflex consonants in $/ \mathrm{i} /$ context.

\section{Discussion \& Conclusion}

Our results show that although speakers are able to achieve constriction at a fairly stable location in the postalveolar region of the palate across vowel contexts, the maneuvering necessary to achieve that constriction is variable. The degree of retroflexion during the production of so-called retroflex consonants, and even whether retroflexion was present at all, varied according to whether they were produced in the high front $/ \mathrm{i} /$ context or the back $/ \mathrm{a} /$ and $/ \mathrm{u} /$ contexts. The presence of negative curvature, corresponding to tongue curling, during both stops and rhotics in back vowel contexts contrasts with what we see during the production of consonants in the /i/ context: either positive curvature scores or negative curvature scores of a low magnitude. It seems that in this vowel context, backing of the tongue tip necessary for post-alveolar constriction is achieved through bunching of the tongue rather than curling.

The variability in tongue shaping during retroflex consonants in Tamil indicates that there is little global control over the tongue during their production. Rather, in keeping with the task dynamic model of speech production [9], the specified task for retroflex consonants is simply post-alveolar constriction. The articulatory maneuvering necessary for achieving this constriction will vary with the state of the vocal tract created by the surrounding vowel context. The consonantal constriction task appears to be superimposed over the tasks controlling the shaping and position of the tongue body for the surrounding vowels, in keeping with previous work [10]. In some cases, articulatory maneuvering will involve retroflexion of the tongue, from which this class of consonants derive their name, but in other cases maneuvering will involve tongue bunching.

A possible avenue for future work is comparison of Tamil's apparently constriction-based production goal with the production goals of other languages that include retroflexes in their inventories. Particularly interesting would be a comparison with a language such as Arrernte (spoken in Australia), which is said to contrast apical and laminal postalveolar consonants [1]. In such a case, it would appear that tongue posture alone distinguishes these two consonants, and as such we might expect less variation in tongue shaping for these consonants across vowel contexts. If examination of the 
two post-alveolar consonants of Arrernte turns up what looks like a tongue-shaping goal of production, it could serve as an interesting contrast to the Tamil retroflex's constriction-based production goal. Such a contrast could be an interesting contribution to the discussion of cross-linguistic differences in the implementation of retroflex consonants.

\section{Acknowledgments}

This work was funded through NIH Grant DC007124 and NSF Grant 1246750 to the University of Southern California.

\section{References}

[1] P. Ladefoged and I. Maddieson, The Sounds of the World's Languages. Blackwell Publishing, 1996.

[2] E. Keane, "Tamil," Journal of the International Phonetic Association, vol. 34, no. 1, pp. 111-116, Jan. 2004.

[3] P. Ladefoged and P. Bhaskararao, "Non-quantal aspects of consonant production: A study of retroflex consonants," Journal of Phonetics, vol. 11, pp. 291-302, 1983.

[4] C. Wiltshire and L. Goldstein, "Tongue Tip Orientation and Coronal Consonants," in Proceedings of the Eastern States Conference on Linguistics, 1997.

[5] S. Narayanan, D. Byrd, and A. Kaun, "Geometry, kinematics, and acoustics of Tamil liquid consonants," The Journal of the Acoustical Society of America, vol. 106, no. 4, pp. 1993-2007, Oct. 1999.

[6] S. Narayanan, K. Nayak, S. Lee, A. Sethy, and D. Byrd, "An approach to real-time magnetic resonance imaging for speech production," Journal of the Acoustical Society of America, vol. 115, no. 4, pp. 1771-1776, 2004.

[7] S. Narayanan, E. Bresch, P. K. Ghosh, L. Goldstein, A. Katsamanis, Y. Kim, A. Lammert, M. I. Proctor, V. Ramanarayanan, and Y. Zhu, "A multimodal real-time MRI articulatory corpus for speech research," in Proceedings of the International Conference on Speech Communication and Technology, 2011.

[8] M. I. Proctor, D. Bone, and S. Narayanan, "Rapid semiautomatic segmentation of real-time Magnetic Resonance Images for parametric vocal tract analysis," in Proceedings of the International Conference on Speech Communication and Technology, 2010.

[9] E. L. Saltzman and K. G. Munhall, “A Dynamical Approach to Gestural Patterning in Speech Production," Ecological Psychology, vol. 1, no. 4, pp. 333-382, 1989.

[10] S. E. G. Öhman, "Coarticulation in VCV Utterances: Spectrographic Measurements," Journal of the Acoustical Society of America, vol. 39, no. 1, pp. 19-36, 1966. 